\title{
An optimized molecular inclusion complex of diferuloylmethane: enhanced physical properties and biological activity
}

This article was published in the following Dove Press journal:

International Journal of Nanomedicine

8 October 2012

Number of times this article has been viewed

\section{Qunyou Tan ${ }^{1, *}$ \\ Yi Li ${ }^{2-4, *}$ \\ Jianyong $\mathrm{Wu}^{2, *}$ \\ $\mathrm{Hu} \mathrm{Mei}^{5}$ \\ Chunjing Zhao ${ }^{3}$ \\ Jingqing Zhang ${ }^{2}$}

'Department of Thoracic Surgery, Institute of Surgery Research, Daping Hospital, Third Military Medical University, ${ }^{2}$ Medicine Engineering Research Center, Chongqing Key Laboratory of Biochemical and Molecular Pharmacology, Chongqing Medical University, ${ }^{3}$ School of Pharmacy, Second Affiliated Hospital, Chongqing Medical University, ${ }^{4}$ School of Pharmacy, The First People's Hospital of Chongqing, ${ }^{5}$ College of Bioengineering, Chongqing University, Chongqing, People's Republic of China

*These authors contributed equally to this work
Correspondence: Jingqing Zhang Medicine Engineering Research Center, Chongqing Key Laboratory of Biochemical and Molecular Pharmacology, Chongqing Medical University, Room 50I, Building 22, Jingdi Garden, 6 Dahuang Road, Yuzhong District, Chongqing 400042, People's Republic of China Tel +86 I3308300303

Fax +862368767988

Email zjqrae0I@।63.com
Objective: The purpose of this study was to explore and evaluate the enhanced physical properties and biological activity of a molecular inclusion complex (MICDH) comprising diferuloylmethane (DFM) and hydroxypropyl- $\beta$-cyclodextrin.

Methods: The preparation conditions of MICDH were optimized using an orthogonal experimental design. The solubility, in vitro release and model fitting, microscopic morphology, molecular structure simulation, anti-lung cancer activity, and action mechanism of MICDH were evaluated.

Results: The solubility of DFM was improved 4400-fold upon complexation with hydroxypropyl$\beta$-cyclodextrin. The release rate of DFM was significantly higher from MICDH than from free DFM. MICDH exhibited higher antitumor activity against human lung adenocarcinoma A549 cells than free DFM. More cells were arrested in the S/G2 phase of the cell cycle or were induced to undergo apoptosis when treated with MICDH than when treated with free DFM. Furthermore, increased reactive oxygen species and intracellular calcium ion levels and decreased mitochondrial membrane potential were observed in cells treated with MICDH.

Conclusion: MICDH markedly improved the physical properties and antitumor activity of DFM. MICDH may prove to be a preferred alternative to free DFM as a formulation for DFM delivery in lung cancer treatment.

Keywords: biological properties, action mechanism, hydroxypropyl- $\beta$-cyclodextrin, antitumor activity

\section{Introduction}

Lung cancer is a very common cancer associated with high morbidity and mortality rates. Every year worldwide over 1.6 million people are diagnosed with lung cancer and over 1.3 million patients die from this disease. ${ }^{1}$ There is an urgent need to develop new therapeutic drugs and new drug delivery systems with higher activity against lung cancer and lower side effects than clinically used drugs. Diferuloylmethane (DFM), a natural polyphenolic extract of turmeric, definitely shows inhibitory activity against many cancer cells, including those of the lung, liver, stomach, ovaries, breast, bladder, head, and neck. ${ }^{2,3}$ Meanwhile, DFM has shown almost no toxicity against normal cells and dose-limiting toxicity in all animal tests and human clinical trials. ${ }^{4}$ It seems very reasonable, then, that DFM is regarded as one of the most promising antitumor drugs, even though it has not been available clinically so far. The clinical translation and application of DFM may be severely confined, mainly by its very low solubility.

Molecular inclusion complexes often refer to special complex compounds in which the molecules of one component (guest molecules) are contained wholly or 
partially within the cavity structure of the other component (host molecules). The cavity nature (a hydrophilic opening and a hydrophobic interior) of the molecules of cyclodextrin or their derivative (the most frequently used host molecules) defines the way in which these molecules form complexes with low-molecular-weight hydrophobic drugs. Although molecular inclusion complex technology is already used to increase the solubility of different types of lipophilic drugs ${ }^{5,6}$ and the bioactivities (such as ocular anti-inflammatory, ${ }^{7}$ anticervical cancer, ${ }^{8}$ antibreast cancer, ${ }^{9}$ antileukemia, ${ }^{10}$ and antiepileptic activities ${ }^{11}$ ) of a few drugs, so far only a few studies have employed it to improve the antilung cancer activity of lipophilic drugs. That is to say, further efforts need to be made before the potential for a molecular inclusion complex to be a good therapeutic antilung cancer drug delivery system can be judged. Furthermore, considering that hydroxypropyl- $\beta$-cyclodextrin (HBCD) has better biocompatibility and higher water solubility than cyclodextrin or other cyclodextrin derivatives, ${ }^{12}$ a molecular inclusion complex consisting of DFM and HBCD (MICDH) is worth exploring. MICDH may have higher solubility than, and enhanced antilung cancer activity compared with, free DFM.

In the experiments outlined in this article, an optimized formulation of MICDH and the optimal process parameters for the preparation of MICDH were investigated. The aim of this study was to explore and evaluate the enhanced physical properties and antitumor activity of MICDH, including its solubility, in vitro release and model fitting, microscopic morphology, molecular structure simulation, antilung cancer activity, and action mechanisms.

\section{Materials and methods}

Shaanxi Sciphar Biotechnology Co, Ltd (Xi'an, China) provided the DFM. HBCD was purchased from Xinxin Pharmaceutical Excipients Co, Ltd (Taixing, China). Roswell Park Memorial Institute 1640 medium containing 10\% fetal bovine serum was purchased from Life Technologies (Carlsbad, CA). Methylthiazol tetrazolium, propidium iodide, and ribonuclease A were purchased from Sigma-Aldrich (St Louis, MO). Fluo-3 AM, DCFH, and rhodamine 123 were purchased from Beyotime Institute of Biotechnology (Shanghai, China). All other reagents and solvents used in this study were of analytical grade.

\section{Preparation and optimization of $\mathrm{MICDH}$}

Weighted HBCD and DFM were mixed in a mortar. The mixture was kneaded, with an appropriate amount of distilled water intermittently added during this kneading process until the mixture formed a slurry. The mixture was then placed in a water bath, where the reaction temperature was controlled and the mixture became a paste. The paste was then placed in a vacuum drying oven at $35^{\circ} \mathrm{C}$ for 3 hours and then in a desiccator chamber at $20^{\circ} \mathrm{C}$ for another 24 hours. Finally, the dried paste was crushed into a powder. ${ }^{13}$

Results of the preliminary experiments revealed that the factors of the DFM-to-HBCD molar ratio (mol:mol), preparation temperature (in degrees Celsius), and kneading time (in hours) had a relatively strong influence on the solubility. Thus, a three-factor, three-level orthogonal experimental design was used to find the optimal levels of each factor (Table 1). ${ }^{14}$ In order to examine the influence of the complex formulation on the solubility, various formulations of $\mathrm{MICDH}$ were prepared under different preparation conditions according to the orthogonal design.

DFM concentrations were determined with an ultravioletvisible spectrophotometer (UV-3150; Shimadzu Corporation, Kyoto, Japan). As shown in Figure 1, HBCD served as a blank reference and did not interfere with the determination of DFM. The calibration curve was linear over the range of $1.1-5.5 \mu \mathrm{g} / \mathrm{mL}(\mathrm{A}=0.1493 \mathrm{C}+0.0058 ; \mathrm{r}=0.9999 ; \mathrm{n}=7$; "A" refers to the absorbance of DFM at $425 \mathrm{~nm}$, and " $\mathrm{C}$ " refers to the concentration of DFM in absolute ethanol). The intraday and interday relative standard derivation ranged from $0.35 \%$ to $1.23 \%$. The mean recovery of DFM was $99.89 \% \pm 0.41 \%(\mathrm{n}=9)$. The ultraviolet-visible method provided an assay that was both sensitive and specific for quantifying DFM.

Table I Results of orthogonal design $(n=3)$

\begin{tabular}{lllll}
\hline $\begin{array}{lllll}\text { Experiment } \\
\text { number }\end{array}$ & \multicolumn{2}{l}{ Factor } & & $\begin{array}{l}\text { Mean solubility } \\
\text { (mmol/L) }\end{array}$ \\
\cline { 2 - 4 } & A & B & C & \\
\hline $\mathrm{I}$ & $\mathrm{I}: \mathrm{I}$ & 20 & 0.5 & $\mathrm{I} .08$ \\
2 & $\mathrm{I}: \mathrm{I}$ & 40 & $\mathrm{I}$ & $\mathrm{I} .49$ \\
3 & $\mathrm{I}: \mathrm{I}$ & 60 & $\mathrm{I} .5$ & $\mathrm{I} .76$ \\
4 & $\mathrm{I}: 2$ & 20 & $\mathrm{I} .5$ & 0.85 \\
5 & $\mathrm{I}: 2$ & 40 & 0.5 & $\mathrm{I} .55$ \\
6 & $\mathrm{I}: 2$ & 60 & $\mathrm{I}$ & 0.83 \\
7 & $\mathrm{I}: 3$ & 20 & $\mathrm{I}$ & 0.76 \\
8 & $\mathrm{I}: 3$ & 40 & $\mathrm{I} .5$ & 0.4 \\
9 & $\mathrm{I}: 3$ & 60 & 0.5 & 0.28 \\
$\mathrm{k}_{1}$ & $\mathrm{I} .44$ & 0.90 & 0.77 & - \\
$\mathrm{k}_{2}$ & $\mathrm{I} .08$ & $\mathrm{I} . \mathrm{I} 5$ & 0.87 & - \\
$\mathrm{k}_{3}$ & 0.48 & 0.96 & $\mathrm{I} .36$ & - \\
$\Delta \mathrm{k}$ & 0.96 & 0.25 & 0.59 & - \\
\hline
\end{tabular}

Notes: "A" represents the molar ratio of diferuloylmethane to hydroxypropyl- $\beta$ cyclodextrin; " $B$ " represents the optimal preparation temperature; "C" represents the optimal kneading time; $k_{i}$ is the average scores of each factor at its different level $i$ (here, i equals I, 2 , and 3 , respectively). 


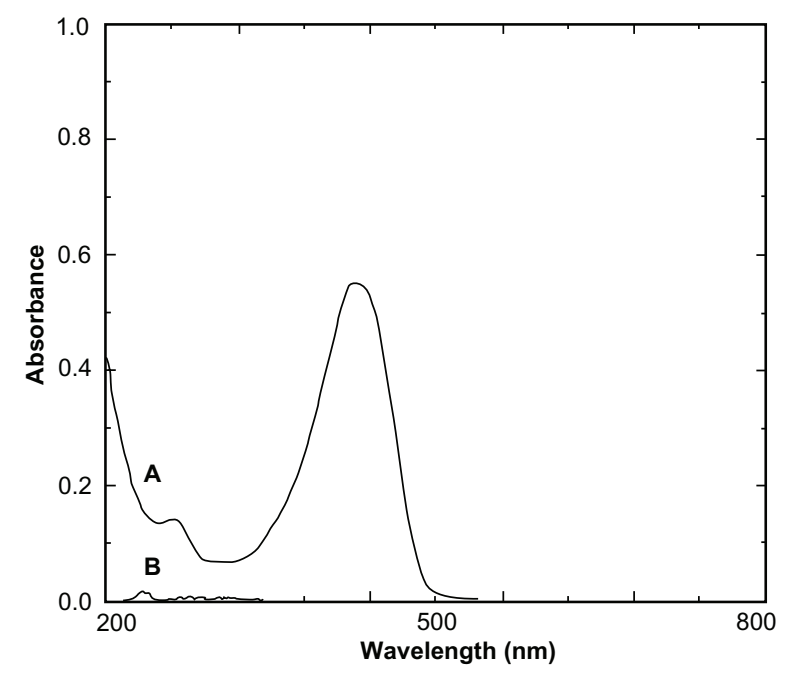

Figure I Ultraviolet spectra of diferuloylmethane (A) and hydroxypropyl- $\beta$ cyclodextrin (B).

\section{Morphology and structure}

The morphology and the structure of all powder samples (DFM, HBCD, physical mixture, and MICDH) were separately investigated using biomicroscopy (XSP-35-1600X; Phoenix, Shangrao, China), and photomicrographs were taken at suitable magnifications (C-60 Zoom; Olympus Corporation, Tokyo, Japan). The schematic structure of MICDH was further simulated according to the DFM-HPCD molecular inclusion complex with 1:1 stoichiometry, using stick and space-filling models.

\section{In vitro release rate studies}

The studies were performed using a modified dialysis method. ${ }^{15}$ Briefly, MICDH containing $10 \mathrm{mg}$ of DFM was loaded into dialysis bags and these were soaked in the release medium of either $0.1 \mathrm{~mol} / \mathrm{L}$ hydrochloric acid $(\mathrm{HCl})$ or $\mathrm{pH} 6.8$ phosphate-buffered saline (PBS) containing $2 \%$ sodium dodecyl sulfate (SDS). ${ }^{13,16}$ This dissolution study was run at $100 \mathrm{rpm}$, and the dissolution vessel was maintained at a mean temperature of $37^{\circ} \mathrm{C} \pm 0.5^{\circ} \mathrm{C}$. Aliquots $(1.0 \mathrm{~mL})$ of the sample were withdrawn at designated time intervals. To maintain a constant volume, the release medium taken away was replaced by $1.0 \mathrm{~mL}$ of fresh release medium. The quantitative determination of DFM was performed with a spectrophotometer and the cumulative release rate was then calculated. The procedures were applied to three batches of MICDH and free DFM. ${ }^{16}$

The classic similarity factor $\left(f_{2}\right)$ method was chosen to evaluate the similarity between two release profiles. The $f_{2}$ value was calculated according to the following equation (where $f_{2}$ was the similarity factor, $\overline{X_{i i}}$ and $\overrightarrow{X_{r i}}$ were the cumulative amount of drug released at time $t$ of two release profiles, $\mathrm{n}$ was the number of sampling points, and $W_{t}$ was the weight), Equation (1):

$$
f_{2}=50 \lg \left\{\left[1+(1 / n) \sum_{i=1}^{n} W_{t}\left(\overline{X_{i i}}-\overrightarrow{X_{r i}}\right)^{2}\right]^{-1 / 2} \times 100\right\}
$$

The $f_{2}$ value could be classified into one of the following levels: (1) equal to 100 , indicating two release curves were almost exactly the same; (2) between 50 and 100, indicating that the similarity between two release curves was statistically significant; and (3) lower than 50, indicating that the similarity was not statistically significant. ${ }^{17}$

\section{Antitumor activity of MICDH}

Human lung adenocarcinoma A549 cells were seeded at a density of $1 \times 10^{4}$ cells per well in 96-well plates for 24 hours before the culture media were replaced with fresh media containing $10 \mu \mathrm{g} / \mathrm{mL}$ of MICDH. After 24 hours, the media were exchanged with fresh media containing $5 \mathrm{mg} / \mathrm{mL}$ methylthiazol tetrazolium solution. The absorbance values were then measured at $570 \mathrm{~nm}$ with a microplate photometer (Sunrise; Tecan Trading AG, Salzburg, Austria). Furthermore, A549 cells cultured in $25 \mathrm{~cm}^{2}$ flasks were treated with $10 \mu \mathrm{g} / \mathrm{mL}$ of MICDH for 24 hours. Cells were harvested by trypsinization and centrifugation and were then fixed with $70 \%$ ethanol at $4^{\circ} \mathrm{C}$ overnight. After being rinsed twice with PBS, cells were resuspended in a DNA-staining solution containing $40 \mu \mathrm{g} / \mathrm{mL}$ propidium iodide and $0.1 \mathrm{mg} / \mathrm{mL}$ ribonuclease $\mathrm{A}$ at $25^{\circ} \mathrm{C}$ for 30 minutes. The cell cycle was analyzed with a FACSVantage flow cytometer (Becton, Dickinson and Company, San Jose, CA) equipped with CellQuest software (Becton, Dickinson and Company). Apoptotic cell quantification was determined using an Annexin V-FITC/PI apoptosis detection kit (Tianjin Sungene Biotech Co, Ltd, Tianjin, China). A549 cells treated with $10 \mu \mathrm{g} / \mathrm{mL}$ of MICDH for 24 hours were collected, centrifuged, and resuspended in a staining solution containing one kind of $5 \mu \mathrm{mol} / \mathrm{L}$ fluorescent dye (DCFH, Fluo-3 AM, or rhodamine 123 ) at $37^{\circ} \mathrm{C}$ for 30 minutes. The reactive oxygen species (ROS) and intracellular free calcium ion $\left(\mathrm{Ca}^{2+}\right)$ levels and the mitochondrial membrane potential were then analyzed using the FACSVantage flow cytometer.

\section{Statistical analysis}

Unless otherwise specified, all data are shown as the mean plus or minus the standard deviation. One-way analysis of variance, Scheffé's $F$-test, and Student's $t$-test were used 
for statistical analysis to determine significant differences. A statistical significance was established at $P<0.05$. Analyses were performed using the StatView statistical package (v 5.0; SAS Institute, Inc, Cary, NC). ${ }^{18}$

\section{Results}

\section{Optimized formulation and preparation of $\mathrm{MICDH}$}

In this study, the optimal levels of each factor were found to be $\mathrm{A}_{1} \mathrm{~B}_{2} \mathrm{C}_{3}$; that is, the optimal values for the DFM-toHBCD molar ratio (represented by $A$ ), the preparation temperature (in degrees Celsius and represented by B), and the kneading time (in hours and represented by $\mathrm{C}$ ) were found to be $1: 1,40^{\circ} \mathrm{C}$, and 1.5 hours, respectively (see Table 1). The solubility of MICDH prepared under the optimal protocol described was recorded as $1.76 \pm 0.03 \mathrm{mg} / \mathrm{mL}$. MICDH was much more soluble than free DFM (40 ng/mL; $\mathrm{n}=3)$.

\section{Characterization of $\mathrm{MICDH}$}

As shown in Figure 2, under the optical microscope, the MICDH powders (ie, the MICDH aggregates) appeared irregular and amorphous, and they were much larger than the free DFM or HBCD aggregates, especially the former. The HBCD aggregates appeared spherical in shape, while the physical mixture showed simple additive effects of free DFM and HBCD.

Figure 3 shows the schematic structure of $\mathrm{MICDH}$ simulated according to the DFM-HBCD complex with 1:1 stoichiometry, using stick and space-filling models.
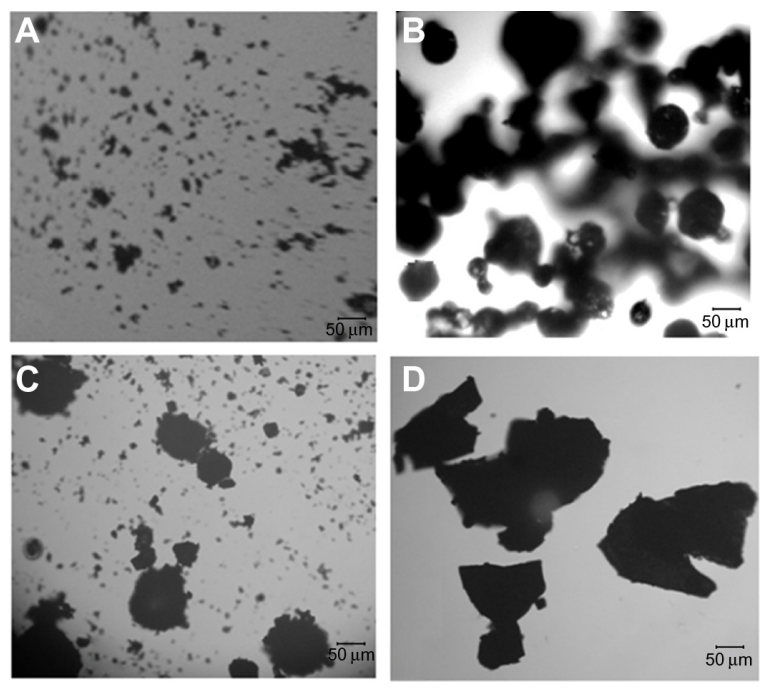

Figure 2 Optical photomicrographs of (A) diferuloylmethane, (B) hydroxypropyl- $\beta$ cyclodextrin, (C) physical mixture, and (D) a molecular inclusion complex consisting of diferuloylmethane and hydroxypropyl- $\beta$-cyclodextrin.
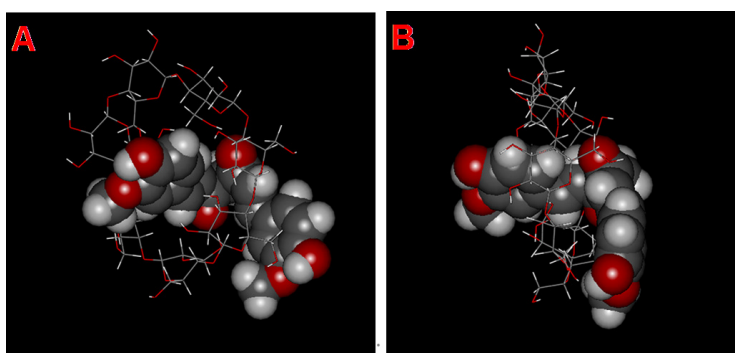

Figure 3 Schematic structure models of a molecular inclusion complex consisting of diferuloylmethane (DFH) and hydroxypropyl- $\beta$-cyclodextrin (HBCD). (A) Initial view and $(\mathbf{B})$ rotated view.

Notes: DFH is represented as a space-filling model, while HBCD is represented as a stick model. The DFH and HBCD molecules consist of different atoms of oxygen (red), carbon (gray), and hydrogen (white).

\section{In vitro release studies}

The drug release profiles of MICDH and free DFM containing the same amount of DFM in two release media (0.1 mol/L HCL and pH 6.8 PBS containing 2\% SDS) are shown in Figure 4. The release rates of DFM from MICDH increased obviously compared with those from free DFM in every release medium. The cumulative release rates for $\mathrm{MICDH}$ in the two release media over 10 hours were more than $35 \%$, while the cumulative release rates for free DFM were zero. Furthermore, rates of MICDH and free DFM were $\sim 55 \%$ versus (vs) $\sim 10 \%$ in 48 hours, and $\sim 80 \%$ vs $<30 \%$ in 120 hours, respectively. As shown in Table 2, several mathematical models were used to fit the MICDH release data. ${ }^{17}$ The results suggest that both the first-order kinetic model and the Higuchi model could be used to fit the release data well in the release media of $0.1 \mathrm{~mol} / \mathrm{L} \mathrm{HCl}$ and $\mathrm{pH} 6.8 \mathrm{PBS}$ containing $2 \%$ SDS.

Different $f_{2}$ values indicated whether two release profiles shared statistically significant similarity. When the value was

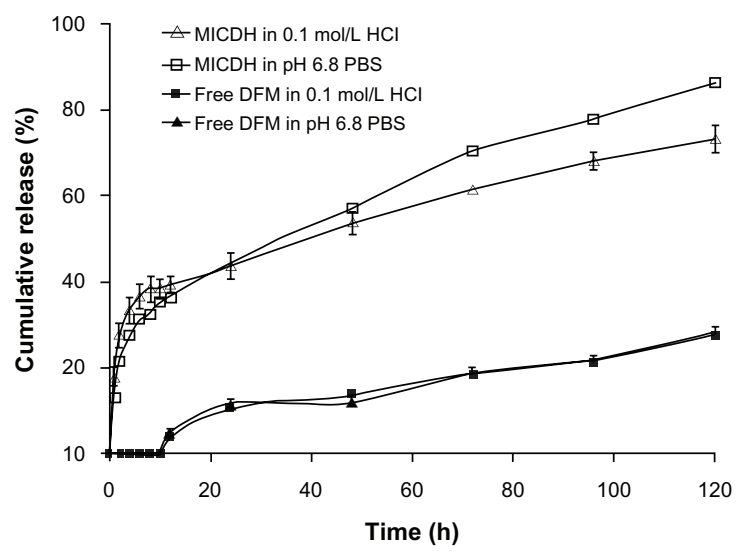

Figure 4 Drug release profiles of different diferuloylmethane (DFM) formulations in $0.1 \mathrm{~mol} / \mathrm{L}$ hydrochloric acid $(\mathrm{HCl})$ and $\mathrm{pH} 6.8$ phosphate-buffered saline (PBS) containing $2 \%$ sodium dodecyl sulfate. 
Table 2 Mathematical models of mean cumulative release rate versus time

\begin{tabular}{|c|c|c|c|c|}
\hline \multirow[t]{2}{*}{ Model } & \multicolumn{2}{|l|}{ MICDH } & \multicolumn{2}{|l|}{ Free DFM (12-I 20 hours) } \\
\hline & $0.1 \mathrm{~mol} / \mathrm{L} \mathrm{HCl}$ & pH 6.8 PBS & $0.1 \mathrm{~mol} / \mathrm{L} \mathrm{HCl}$ & pH 6.8 PBS \\
\hline Zero-order & $Q=0.3842 t+31.47 \mid$ & $Q=0.5512 t+26.117$ & $Q=0.1954 t+4.0409$ & $Q=0.197 t+4.0818$ \\
\hline kinetic & $r=0.9480$ & $r=0.9692$ & $r=0.9818$ & $r=0.9786$ \\
\hline First-order & $\ln (I-Q)=-0.0082 t-0.360$ & $\ln (I-Q)=-0.014 t-0.244$ & $\ln (I-Q)=-0.0023 t-0.0348$ & $\ln (I-Q)=-0.0024 t-0.0346$ \\
\hline kinetic & $r=0.9832$ & $r=0.9947$ & $r=0.9850$ & $r=0.9799$ \\
\hline \multirow[t]{2}{*}{ Higuchi ${ }^{17}$} & $Q=4.7755 t^{1 / 2}+21.537$ & $Q=0.1459 t^{1 / 2}-1.7299$ & $Q=2.849 \mid t^{1 / 2}-4.9889$ & $Q=2.8418 t^{1 / 2}-4.7928$ \\
\hline & $r=0.9822$ & $r=0.9956$ & $r=0.9857$ & $r=0.9718$ \\
\hline Retrer- & $\ln Q=0.2521 \ln t+3.064$ & $\ln Q=0.3555 \ln t+2.735$ & $\ln Q=0.7487 \ln t-0.2637$ & $\ln Q=0.6918 \ln t-0.0217$ \\
\hline Peppas $^{33}$ & $r=0.9798$ & $r=0.9921$ & $r=0.9742$ & $r=0.9673$ \\
\hline \multirow[t]{3}{*}{ Weibull17 $^{17}$} & $\ln \ln [1 /(I-Q)]=0.3438$ & $\ln \ln [1 /(1-Q)]=0.4972$ & $\ln \ln [1 / 1-Q]=0.8016$ & $\ln \ln [1 / I-Q]=0.744 \mathrm{I}$ \\
\hline & $\ln t-1.4846$ & $\ln t-1.9177$ & $\ln t-4.9844$ & $\ln t-4.7398$ \\
\hline & $r=0.9825$ & $r=0.9872$ & $r=0.9767$ & $r=0.9682$ \\
\hline
\end{tabular}

Note: $Q$ is cumulative DFM release at time $t$.

Abbreviations: MICDH, molecular inclusion complex consisting of diferuloylmethane and hydroxypropyl- $\beta$-cyclodextrin; DFM, diferuloylmethane; HCl, hydrochloric acid; PBS, phosphate-buffered saline.

above 50, the answer was yes; otherwise, the answer was no. As shown in Table 3, the $f_{2}$ values between two release curves in $\mathrm{pH}$ 6.8 PBS and $0.1 \mathrm{~mol} / \mathrm{L} \mathrm{HCl}$ of $\mathrm{MICDH}$ or free DFM were above 50 ( $\sim 59$ and $\sim 96$, respectively), while the $f_{2}$ values between two release curves of MICDH and free DFM in $\mathrm{pH} 6.8 \mathrm{PBS}$ or $0.1 \mathrm{~mol} / \mathrm{L} \mathrm{HCl}$ were much lower than 50 $\left(\sim 21\right.$ and $\sim 22$, respectively). Different $f_{2}$ values clearly show different relationships between the two release profiles.

\section{Antitumor activities of MICDH}

As shown in Figure 5, both MICDH and free DFM inhibited human lung adenocarcinoma A549 cell growth in a dosedependent manner. The mean 50\% inhibitory concentration (the drug concentration causing a 50\% decrease in cell survival) value of MICDH $(8.50 \mu \mathrm{g} / \mathrm{mL})$ decreased by almost a third, compared with that of free DFM $(12.53 \mu \mathrm{g} / \mathrm{mL})$, suggesting that MICDH exhibited a higher antitumor effect than free DFM. Tumor cells treated with $10 \mu \mathrm{g} / \mathrm{mL}$ of either MICDH or free DFM for 24 hours were further analyzed to help better understand the mechanisms involved. As shown in Figure 6, more cells were arrested in the S/G2 phase of the cell cycle ( $\sim 56 \%$ vs $\sim 46 \%)$ or were induced to undergo apoptosis ( $\sim 99 \%$ vs $\sim 55 \%)$ when treated with MICDH than when treated with free DFM. As shown in Figure 7, ROS and intracellular $\mathrm{Ca}^{2+}$ levels in cells treated with $\mathrm{MICDH}$ were much higher than in those treated with free DFM $(P<0.05)$, while the mitochondrial membrane potential in cells treated with MICDH was only a little lower than in those treated with free DFM $(P>0.05)$.

\section{Discussion}

According to statistics, $\sim 70 \%$ of new drug candidates and $\sim 40 \%$ of oral drugs on the market are poorly soluble in water. ${ }^{19}$ The formulation of poorly soluble drugs for oral delivery has always presented a big challenge. Poor aqueous solubility often leads to poor bioavailability and low bioactivity, so the first and most important point to be addressed for the practically insoluble drug DFM was the development of a suitable DFM formulation with better solubility than free DFM. Theoretically, the solubility value should be as high as possible. As far as the methods to improve the solubility of low-solubility drugs are concerned, the molecular inclusion complexation technology can be as effective as other approaches - such as crystal modification, self-emulsification, and nanogranulation - or more so, while also being much simpler and easier for production scale-up. ${ }^{20-22}$

Table 3 The similarities between the two release profiles of a molecular inclusion complex, consisting of diferuloylmethane (DFM) and hydroxypropyl- $\beta$-cyclodextrin $(\mathrm{MICDH})$, and of free DFM in two release media, either the same or different

\begin{tabular}{|c|c|c|c|c|}
\hline \multicolumn{2}{|c|}{ Release profile I } & \multicolumn{2}{|c|}{ Release profile 2} & \multirow{2}{*}{$\begin{array}{l}\text { Similarity } \\
\text { factor }\left(f_{2}\right)\end{array}$} \\
\hline Formulation & Release medium & Formulation & Release medium & \\
\hline $\mathrm{MICDH}$ & $0.1 \mathrm{~mol} / \mathrm{L} \mathrm{HCl}$ & Free DFM & $0.1 \mathrm{~mol} / \mathrm{L} \mathrm{HCl}$ & 21.53 \\
\hline $\mathrm{MICDH}$ & $\mathrm{pH} 6.8$ PBS & Free DFM & $\mathrm{pH} 6.8$ PBS & 20.56 \\
\hline $\mathrm{MICDH}$ & $0.1 \mathrm{~mol} / \mathrm{L} \mathrm{HCl}$ & $\mathrm{MICDH}$ & $\mathrm{pH} 6.8$ PBS & 58.86 \\
\hline Free DFM & $0.1 \mathrm{~mol} / \mathrm{L} \mathrm{HCl}$ & Free DFM & $\mathrm{pH} 6.8$ PBS & 96.01 \\
\hline
\end{tabular}

Abbreviations: $\mathrm{HCl}$, hydrochloric acid; PBS, phosphate-buffered saline. 


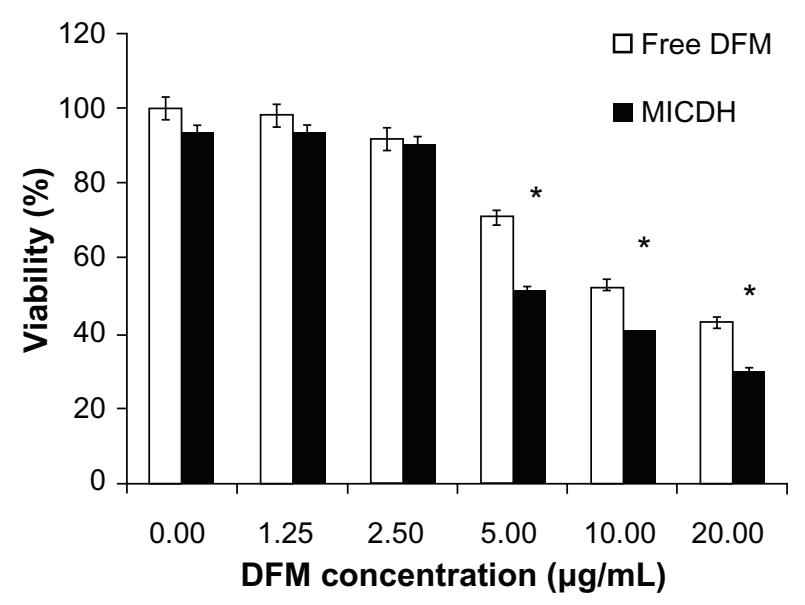

Figure 5 Cell viabilities after 24 hours' exposure to different concentrations of free diferuloylmethane (DFM) and a molecular inclusion complex consisting of DFM and hydroxypropyl- $\beta$-cyclodextrin (MICDH).

Notes: Results are presented as the mean plus or minus the standard deviation; $\mathrm{n}=5$; * $\mathrm{P}<0.05$ for MICDH compared with free DFM.

In the preliminary experiments, several preparation methods (eg, coprecipitation and sonication methods) and the type of host molecules (eg, $\beta$ - and $\alpha$-cyclodextrin) were screened (data not shown). Following this, HBCD were chosen to prepare the MICDH by kneading method. Three critical factors (the DFMto-HBCD molar ratio, the preparation temperature, and the kneading time) that had relatively strong influence on solubility were investigated. An orthogonal design was used to optimize the formulation and preparation process of $\mathrm{MICDH}$ - the orthogonal design, one of the most used designs in medical and pharmaceutical fields, can reflect the principal components associated with each experimental factor. ${ }^{23}$ The formulation with the highest solubility was then obtained. Encouragingly, the solubility of MICDH prepared under optimal conditions was 4400 times that of free DFH. It has been documented that the solubility of brominated noscapine (antiprostate cancer drug) could be improved approximately 11- and 21-fold upon complexation with $\beta$-cyclodextrin and methyl- $\beta$-cyclodextrin, respectively, ${ }^{24}$ while the solubility of 9-nitrocamptothecin (antiliver cancer drug) could be improved 104-fold upon complexation with HBCD. ${ }^{25}$
The formation process of a molecular inclusion complex was a physical rather than a chemical process. A drug molecule was able to enter the host molecular cavity and was held there by intermolecular forces such as van der Waals force and hydrogen bonding rather than covalent bonding. The formation of a molecular inclusion complex was mainly relative to three-dimensional structures and polarity. ${ }^{26}$ The drug molecule, having a suitable size and shape corresponding to that of the cavity, was usually easy to encapsulate within the host molecule. On the other hand, a guest molecule was able to enter and exit a host cavity in a dynamic process. The stability of a molecular inclusion complex mainly depended on the polarities of the guest and host molecules and on the strength of the intermolecular force. Based on the information outlined here, it was easy to determine why the solubilizing effects of different molecular inclusion complexes were not the same.

As expected, compared with free DFM, the release rates of DFM from MICDH increased obviously in different release media $(0.1 \mathrm{~mol} / \mathrm{L} \mathrm{HCl}$ or $\mathrm{pH} 6.8 \mathrm{PBS}$ containing $2 \% \mathrm{SDS}$ ). The popular $f_{2}$ method was further chosen for the release profile comparison. This $f_{2}$ method, which is recommended by the US Food and Drug Administration to evaluate the similarity between two dissolution profiles, was simple and reliable. As shown in Table 3 , the $f_{2}$ value calculated to be $\sim 59$ suggested that the similarity between two release curves for $\mathrm{MICDH}$ in $\mathrm{pH} 6.8 \mathrm{PBS}$ and $0.1 \mathrm{~mol} / \mathrm{L} \mathrm{HCl}$ was statistically significant. The fact that the release rate in $\mathrm{pH} 6.8$ PBS was higher than the release rate in $0.1 \mathrm{~mol} / \mathrm{L} \mathrm{HCl}$ may be ascribed to the existence of the phenolic hydroxyl group in the DFM and the slow release of DFM from MICDH. On the other hand, since there was a significant difference between two curves when the $f_{2}$ value was below 50, the difference between the curves of MICDH and free DFM in each release medium was statistically significant.

Being an alternative system for the oral delivery of a water insoluble-drug, MICDH could be expected to have better absorption and bioavailability than free DFM because
A

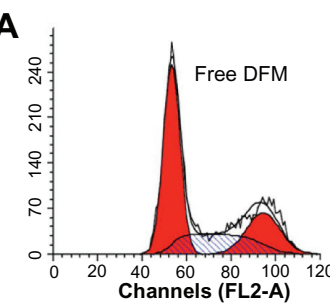

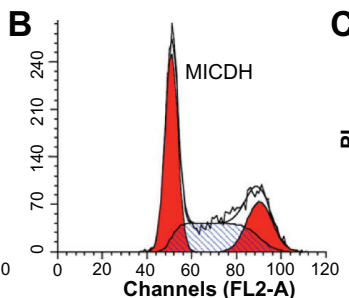
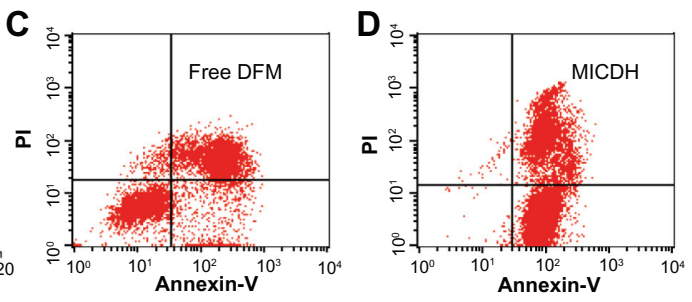

Figure 6 (A and B) Cell cycle arrest and (C and D) cell apoptosis after 24 hours' exposure to $10 \mu g / \mathrm{mL}$ free diferuloylmethane (DFM) and a molecular inclusion complex consisting of DFM and hydroxypropyl- $\beta$-cyclodextrin (MICDH).

Abbreviation: PI, propidium iodide. 

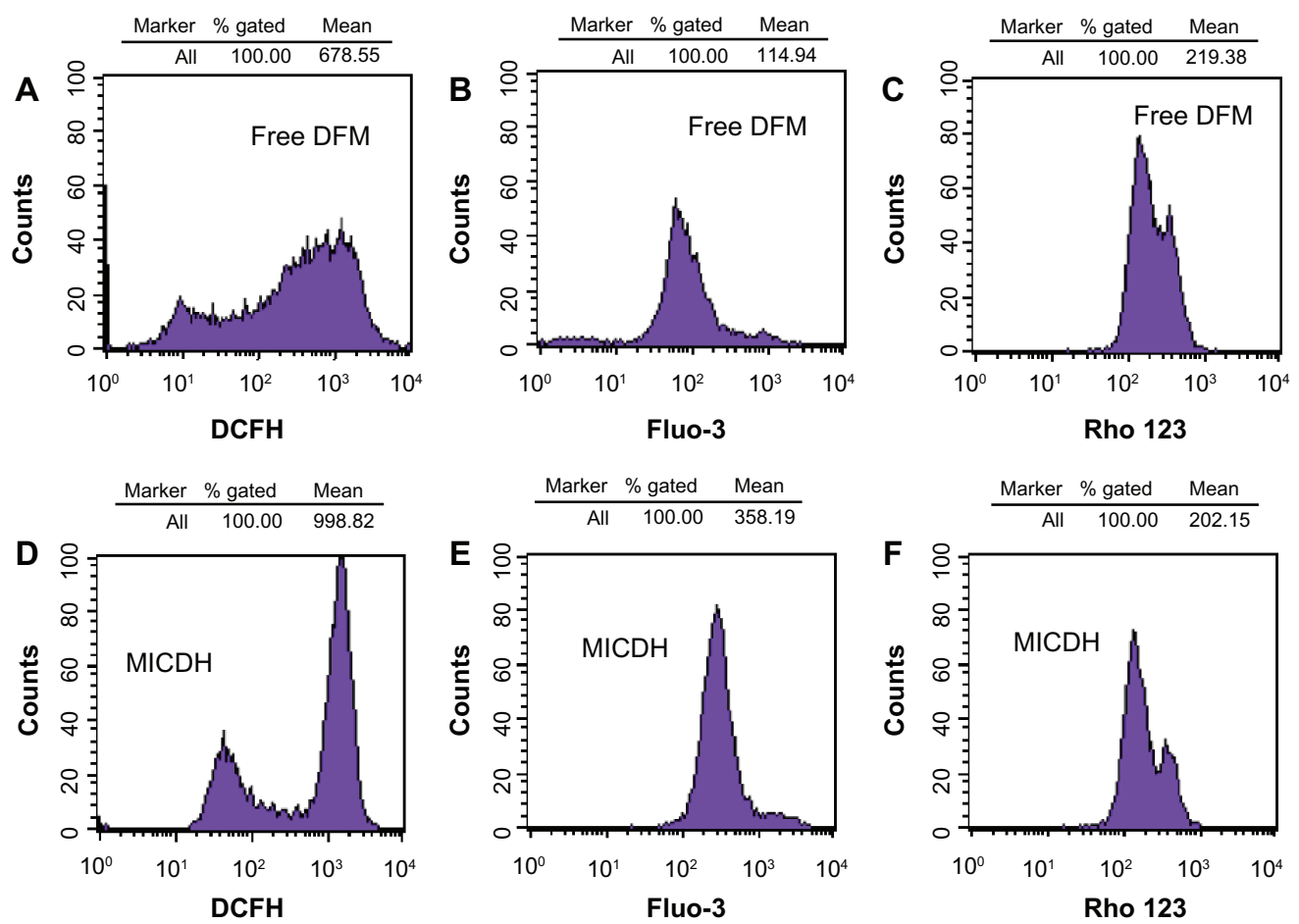

Figure 7 (A and $\mathbf{D})$ The reactive oxygen species levels, (B and $\mathbf{E})$ intracellular free calcium ion levels, and $(\mathbf{C}$ and $\mathbf{F})$ mitochondrial membrane potential after 24 hours' exposure to $10 \mu \mathrm{g} / \mathrm{mL}$ free diferuloylmethane (DFM) and a molecular inclusion complex consisting of DFM and hydroxypropyl- $\beta$-cyclodextrin (MICDH).

Abbreviations: Fluo-3, Fluo-3 AM; Rho I23, rhodamine 123.

of faster release. In clinical practice, this type of formulation may provide a faster, better, and longer duration of anticancer action than that provided by free DFM. The results from this study confirm that the release rates of DFM (a practically water-insoluble drug) could be controlled by molecular inclusion complexation with HBCD (a biodegradable macromolecular excipient). MICDH may be used as a carrier for the enhanced release of DFM in cancer therapy.

Apart from increasing solubility, MICDH improved the antitumor activity against human lung adenocarcinoma A549 cells compared with free DFM. Similar results have been reported in previous studies. ${ }^{11,12}$ For example, the anticancer activity of S1 (a structure-specific B-cell lymphoma-2 inhibitor) against human breast adenocarcinoma MCF-7 cells could be enhanced by complexation with $\gamma$-cyclodextrin; ${ }^{11}$ another example is the anticancer activity against leukemia cells of Bis(tbutyl-S-acyl-2-thioethyl)cytidine monophosophate, which could be enhanced by complexation with HBCD. ${ }^{12}$

The results of this study also showed that MICDH had higher antitumor activity than free DFM. This may be because of the improved solubility and release of $\mathrm{MICDH}$, since MICDH in aqueous solution favored the entrance of DFM into the cell and act. Furthermore, MICDH may inhibit tumor cells in the following two ways. First, MICDH induced
$\mathrm{S} / \mathrm{G} 2$ cell cycle modifications on human lung adenocarcinoma A549 cells; an increased accumulation of cells at the S/G2 phase after MICDH contact was found compared with that after free DFM contact. ${ }^{27}$ Second, incubation of A549 cells with MICDH for 24 hours caused an obvious increase in the proportion of apoptotic cells compared with incubation with free DFM. ${ }^{28}$ The increased induction of apoptosis by MICDH in human lung adenocarcinoma A549 cells, compared with that by free DFM, may be due to the increases of ROS ${ }^{29}$ and intracellular $\mathrm{Ca}^{2+}$ concentration ${ }^{30}$ and the decrease of mitochondrial membrane potential. ${ }^{31}$ A previous study has shown that DFM can inhibit tumor cell growth via different mechanisms other than those mentioned here. ${ }^{32}$ More efforts are needed in regard to understanding the mechanisms responsible for improvement of the antitumor activity of MICDH.

\section{Conclusion}

The results of this study indicate that MICDH enhanced the physical properties and antitumor activity of DFM remarkably. An optimized molecular inclusion complex of DFM was successfully developed to greatly improve the physical properties and antitumor activity of DFM. The solubility of DFM was improved 4400-fold upon complexation with HBCD. The release rates of DFM from 
MICDH were significantly higher than those from free DFM. Preliminary antilung cancer activity and action mechanisms were clarified. $\mathrm{MICDH}$ is a promising alternative to free DRM as a formulation for DFM delivery in lung cancer treatment.

\section{Acknowledgments}

This research was partially supported by grants from the National Natural Science Foundation of China(No 30973645), the Specialized Research Fund for the Doctoral Program of Higher Education (20095503120008), the Chongqing Natural Science Foundation (CSCT2012JJB10027), and the Chongqing Education Committee Fund (the Excellent University Personnel Financial Aid Plan, KJ120321).

\section{Disclosure}

The authors report no conflicts of interest in this work.

\section{References}

1. Siddiqui S, Ali MU, Ali MA, Shah N, Nasreen S. Lung carcinoma: its profile and changing trends. J Ayub Med Coll Abbottabad. 2010;22(3): 116-119.

2. Gupta SC, Sung B, Kim JH, Prasad S, Li S, Aggarwal BB. Multitargeting by turmeric, the golden spice: from kitchen to clinic. Mol Nutr Food Res. Epub August 13, 2012.

3. Wilken R, Veena MS, Wang MB, Srivatsan ES. Curcumin: a review of anti-cancer properties and therapeutic activity in head and neck squamous cell carcinoma. Mol Cancer. 2011;10:12.

4. Jyothi D, Vanathi P, Mangala Gowri P, Rama Subba Rao V, Madhusudana Rao J, Sreedhar AS. Diferuloylmethane augments the cytotoxic effects of piplartine isolated from Piper chaba. Toxicol in Vitro. 2009;23(6):1085-1091.

5. Upreti M, Strassburger K, Chen YL, Wu S, Prakash I. Solubility enhancement of steviol glycosides and characterization of their inclusion complexes with gamma-cyclodextrin. Int J Mol Sci. 2011;12(11): 7529-7553.

6. Zoeller T, Dressman JB, Klein S. Application of a ternary HP- $\beta$-CDcomplex approach to improve the dissolution performance of a poorly soluble weak acid under biorelevant conditions. Int J Pharm. 2012; 430(1-2):176-183.

7. Kanai K, Ito Y, Nagai N, et al. Effects of instillation of eyedrops containing disulfiram and hydroxypropyl- $\beta$-cyclodextrin inclusion complex on endotoxin-induced uveitis in rats. Curr Eye Res. 2012;37(2): 124-131.

8. Azuma H, Aizawa Y, Higashitani N, et al. Biological activity of water-soluble inclusion complexes of 1'-acetoxychavicol acetate with cyclodextrins. Bioorg Med Chem. 2011;19(12):3855-3863.

9. Zhang Z, Wu G, Gao J, Song T. Inclusion complex of a Bcl-2 inhibitor with cyclodextrin: characterization, cellular accumulation, and in vivo antitumor activity. Mol Pharm. 2010;7(4):1348-1354.

10. Diab R, Jordheim LP, Degobert G, et al. Preparation, characterization and in vitro evaluation of a new nucleotide analogue prodrug cyclodextrin inclusion complexes. J Nanosci Nanotechnol. 2009;9(1): 295-300.

11. Jain AS, Date AA, Pissurlenkar RR, Coutinho EC, Nagarsenker MS. Sulfobutyl ether(7) $\beta$-cyclodextrin (SBE(7) $\beta$-CD) carbamazepine complex: preparation, characterization, molecular modeling, and evaluation of in vivo anti-epileptic activity. AAPS Pharm Sci Tech. 2011;12(4):1163-1175.
12. Loftsson T, Brewster ME. Cyclodextrins as functional excipients: methods to enhance complexation efficiency. J Pharm Sci. 2012;101(9): 3019-3032.

13. Tan QY, Liu S, Chen XL, et al. Design and evaluation of a novel evodiamine-phospholipid complex for improved oral bioavailability. AAPS Pharm Sci Tech. 2012;13(2):534-547.

14. Jakobi AJ, Huizinga EG. A rapid cloning method employing orthogonal end protection. PLoS One. 2012;7(6):e37617.

15. Lu B, Zhang JQ, Yang H. Nonphospholipid vesicles of carboplatin for lung targeting. Drug Deliv. 2003;10(2):87-94.

16. Zhang JQ, Liu J, Li XL, Jasti BR. Preparation and characterization of solid lipid nanoparticles containing silibinin. Drug Deliv. 2007;14(6): 381-387.

17. Tan QY, Xu ML, Wu JY, Yin HF, Zhang JQ. Preparation and characterization of poly(lactic acid) nanoparticles for sustained release of pyridostigmine bromide. Pharmazie. 2012;67(4):311-318.

18. Tan QY, Zhang JQ, Wang N, et al. Improved biological properties and hypouricemic effects of uricase from Candida utilis loaded in novel alkaline enzymosomes. Int J Nanomedicine. 2012;7:3929-3938.

19. Kawabata Y, Wada K, Nakatani M, Yamada S, Onoue S. Formulation design for poorly water-soluble drugs based on biopharmaceutics classification system: basic approaches and practical applications. Int J Pharm. 2011;420(1):1-10.

20. Miroshnyk I, Mirza S, Sandler N. Pharmaceutical co-crystals: an opportunity for drug product enhancement. Expert Opin Drug Deliv. 2009;6(4):333-341.

21. Mercuri A, Passalacqua A, Wickham MS, Faulks RM, Craig DQ, Barker SA. The effect of composition and gastric conditions on the self-emulsification process of ibuprofen-loaded self-emulsifying drug delivery systems: a microscopic and dynamic gastric model study. Pharm Res. 2011;28(7):1540-1551.

22. Zhang JQ, Zhang ZR, Yang H, Tan QY, Qin SR, Qiu XL. Lyophilized paclitaxel magnetoliposomes as a potential drug delivery system for breast carcinoma via parenteral administration: in vitro and in vivo studies. Pharm Res. 2005;22(4):573-583.

23. Tiwari S, Singh S, Rawat M, Tilak R, Mishra B. L(9) orthogonal design assisted formulation and evaluation of chitosan-based buccoadhesive films of miconazole nitrate. Curr Drug Deliv. 2009;6(3): 305-316.

24. Madan J, Baruah B, Nagaraju M, et al. Molecular cycloencapsulation augments solubility and improves therapeutic index of brominated noscapine in prostate cancer cells. Mol Pharm. 2012;9(5): 1470-1480.

25. Jiang Y, Jiang X, Law K, et al. Enhanced anti-tumor effect of 9-nitrocamptothecin complexed by hydroxypropyl- $\beta$-cyclodextrin and safety evaluation. Int J Pharm. 2011;415(1-2):252-258.

26. El-Kemary MA, El-Gezawy HS, El-Baradie HY, Issa RM. Spectral and photophysical studies of inclusion complexes of 2-amino-4,6-dimethyl pyrimidine with beta-cyclodextrin. Spectrochim Acta A Mol Biomol Spectrosc. 2002;58(3):493-500.

27. Demarcq C, Bastian G, Remvikos Y. BrdUrd/DNA flow cytometry analysis demonstrates cis-diamminedichloroplatinum (II)-induced multiple cell-cycle modifications on human lung carcinoma cells. Cytometry. 1992;13(4):416-422.

28. Wu J, Zhou J, Lang Y, et al. A polysaccharide from Armillaria mellea exhibits strong in vitro anticancer activity via apoptosis-involved mechanisms. Int J Biol Macromol. 2012;51(4):663-667.

29. Park SH, Kim JH, Chi GY, et al. Induction of apoptosis and autophagy by sodium selenite in A549 human lung carcinoma cells through generation of reactive oxygen species. Toxicol Lett. 2012;212(3): 252-261.

30. Rao YK, Kao TY, Wu MF, Ko JL, Tzeng YM. Identification of small molecule inhibitors of telomerase activity through transcriptional regulation of hTERT and calcium induction pathway in human lung adenocarcinoma A549 cells. Bioorg Med Chem. 2010;18(19): 6987-6994. 
31. Hu W, Kavanagh JJ. Anticancer therapy targeting the apoptotic pathway. Lancet Oncol. 2003;4(12):721-729.

32. Anand P, Sung B, Kunnumakkara AB, Rajasekharan KN, Aggarwal BB. Suppression of pro-inflammatory and proliferative pathways by diferuloylmethane (curcumin) and its analogues dibenzoylmethane, dibenzoylpropane, and dibenzylideneacetone: role of Michael acceptors and Michael donors. Biochem Pharmacol. 2011;82(12):1901-1909.
33. Thakar K, Joshi G, Sawant KK. Bioavailability enhancement of baclofen by gastroretentive floating formulation: statistical optimization, in vitro and in vivo pharmacokinetic studies. Drug Dev Ind Pharm. Epub 2012 Aug 20.

\section{Publish your work in this journal}

The International Journal of Nanomedicine is an international, peerreviewed journal focusing on the application of nanotechnology in diagnostics, therapeutics, and drug delivery systems throughout the biomedical field. This journal is indexed on PubMed Central, MedLine, CAS, SciSearch $®$, Current Contents ${ } /$ Clinical Medicine,
Journal Citation Reports/Science Edition, EMBase, Scopus and the Elsevier Bibliographic databases. The manuscript management system is completely online and includes a very quick and fair peer-review system, which is all easy to use. Visit http://www.dovepress.com/ testimonials.php to read real quotes from published authors.

Submit your manuscript here: http://www.dovepress.com/international-journal-of-nanomedicine-journal 